\title{
Improvement On Thermal Performance Of A Disk-Shaped Miniature Heat Pipe With Nanofluid
}

\author{
Hsin-Tang Chien, Chien-In Tsai, Ping-Hei Chen* and Po-Yeh Chen
}

\author{
Mechanical Engineering Department, National Taiwan University, \\ No. 1, Roosevelt Road Sec. 4, \\ Taipei 10617, Taiwan. \\ Asia Vital Components Co.,Ltd. \\ Tel:886-2-23670781 Fax: 886-2-23631755 \\ Email: phchen@ntu.edu.tw
}

\begin{abstract}
The present study used nanofluid as the working medium for a disk-shaped miniature heat pipe (DMHP). The nanofluid is a suspension with gold nanoparticles of an average diameter of $17 \mathrm{~nm}$ in an aqueous solution. An experimental system was set up to measure the thermal resistance of the disk-shaped miniature heat pipe (DMHP) with both nanofluid and DI-water. A mounting base is designed and integrated with the $D M H P$. as a heat spreader for laser diode TO can package. The present mounting base is made of aluminum (6061 T6). The measured results show that the thermal resistance of DMHP varies with the charge volume and the type of the working medium. At a same charge volume, a significant reduction in thermal resistance of DMHP can be found if nanofluid is used instead of DI-water.
\end{abstract}

KEWORDS: micro groove heat pipe, heat spreader, nanoparticle, thermal enhance, nanofluid

\section{PREPARATION OF NANO-GOLD}

\section{INTRODUCTION}

The demand for low cost and high performance packages is increasing in recent years. One of the choices is to use heat pipe to dissipate the heat generated by CPU and optical components. Many studies have been conducted to reduce the thermal resistance of heat pipe by choosing different working medium and geometrical configuration of microgrooves or meshes. In this study, a nanofluid is used as a working medium in the heat pipe. Fluid with suspended nanoparticles is termed as nanofluid in this study.

Huang et al., 1998[1] showed that heat transfer in pool boiling of water in a heated stainless steel horizontal plate was significantly enhanced by adding glass, copper and stainless steel solid particles of $500 \mu \mathrm{m}$ into the DI water. However, fluids with suspended microparticles are impractical in applications of miniature heat pipes. It is expected that the nanoparticles in working medium can significantly improve the boiling performance of fluid at the evaporator's side of heat pipe. Since nanofluid has better thermal properties than pure fluid in many aspects, the present study aims to improve the thermal performance of DMHP by replacing DI-water with nanofluid.
Gold nanoparticles were synthetized by a citrate reduction of aqueous hydrogen tetrachloroaurate ( $\mathrm{HAuCl} 4)$. An amount of $0.008 \mathrm{mg} \mathrm{HAuCl} 4$ (Sigma Chemical, St. Louis, MO) was dissolved in $80 \mathrm{~mL}$ distilled water as a primer solution. An additional $4 \mathrm{~mL}$ mixture of $3.4 \mathrm{mM}$ citric acid, $0.1 \mathrm{~mL} 5.8 \mathrm{mM}$ tannic acid and $15.9 \mathrm{~mL}$ distilled water were used as a reducing solution. The reducing solution was preheated to $60^{\circ} \mathrm{C}$. After primer solution was heated to the boiling temperature, the reducing solution was added into the primer solution. Figure 1 shows a TEM (Hitachi 8100) image of gold nanoparticles with a diameter of $17 \mathrm{~nm}$ and a concentration was about $17 \mathrm{nM}$.

\section{EXPERIMENTAL MEASUREMENT}

Figures 2(a) and 2(b) respectively show the prototype and a three dimensional view of the present DMHP. Micro-grooves were fabricated on aluminum base by a precise metal forming process. The diameter and thickness of aluminum base are $9 \mathrm{~mm}$ and $2 \mathrm{~mm}$ respectively. The material of the base is aluminum alloy (606! T6). A total number of eighteen micro-grooves were evenly distributed on the aluminum base. The depth and width of micro-grooves are $0.4 \mathrm{~mm}$ and 0.35 mm respectively.

A silicone rubber was sealed on the top of the aluminum base 
with vacuum grease, and the enclosed micro-grooves were charged with working fluid. For the present study, DI water and nanofluid at three different charge volumes with 18,37 . and $55 \%$ of the total void volume were respectively used in the measurement.

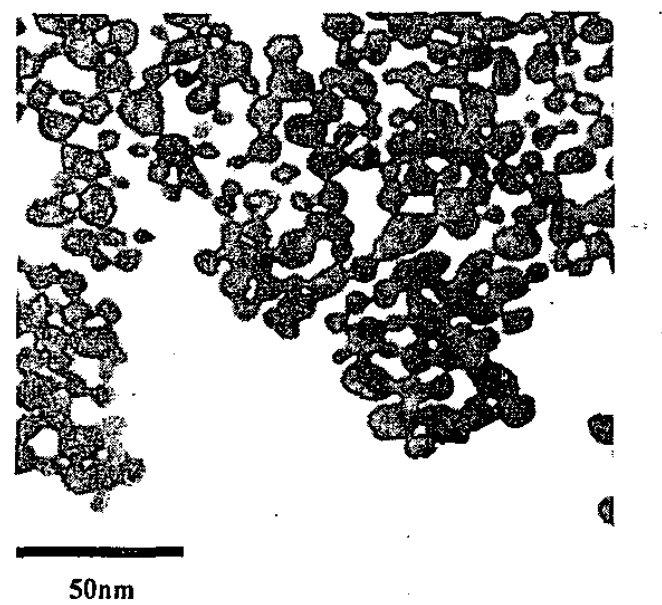

Fig. 1 TEM image of gold nanoparticles with a magnification of 200,000

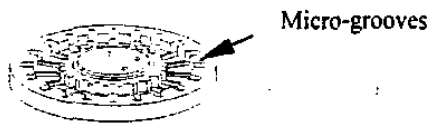

(b) three-dimensional view

Figure 2 The design of DMHP.

A schematic view of the measuring system for the thermal performance of DMHP is shown in Fig. 3. The tested DMHP was fixed on the through hole of a holder. The plexiglas holder was positioned horizontally and had a through hole with a diameter of $8.5 \mathrm{~mm}$. The local temperatures on the DMHP were respectively measured by five thermocouples of type-T. Two thermocouples were attached to the center of aluminum base plate to measure the evaporator's temperature, and three were evenly distributed at the circumference to measure the condenser's temperature. All thermocouples were calibrated against a quartz thermometer. The uncertainty in temperature measurement is about $\pm 0.1^{\circ} \mathrm{C}$. A laser diode with an emission power of $0.35 \mathrm{~W}$ was used as the applied heat source in the measurement. The heating power of laser diode was measured by an optical power meter (Vector $\mathrm{H} 410$, Scientech) with a resolution of $0.001 \mathrm{lW}$. The laser bean was focused on the center of aluminum base where was painted with black paint of 0.95 absorptivity:

Once both the heating load $(Q)$ and the iemperature difference $\left(d T=T_{\text {svopuratar }}-T_{\text {condenser }}\right)$ were measured. the thermal resistance $(R)$ could then be evaluated from the equation, $\dot{R}=d T / Q$.

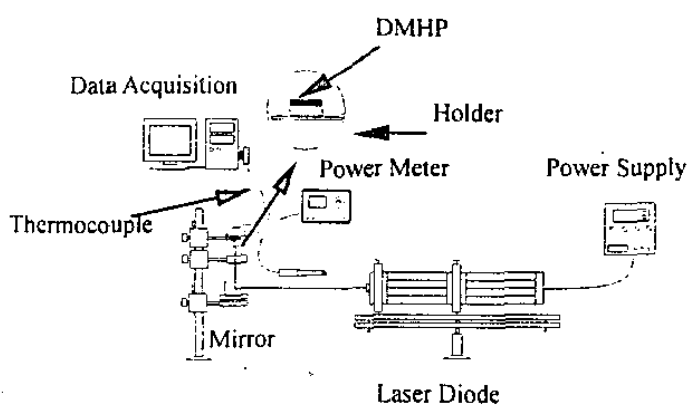

Figure 3 Measuring system.[2]

\section{Results and Conclusion}

The effect of fluid charge volume on the thermal performance of tested DMHP is shown in Fig. 4. As compared DMHP with DI water, the present measured data verify that the proposed DMHP with nano-gold particles have a lower thermal resistance under any charge volume. Meanwhile, the comparison shows that an average decrease of $40 \%$ in the

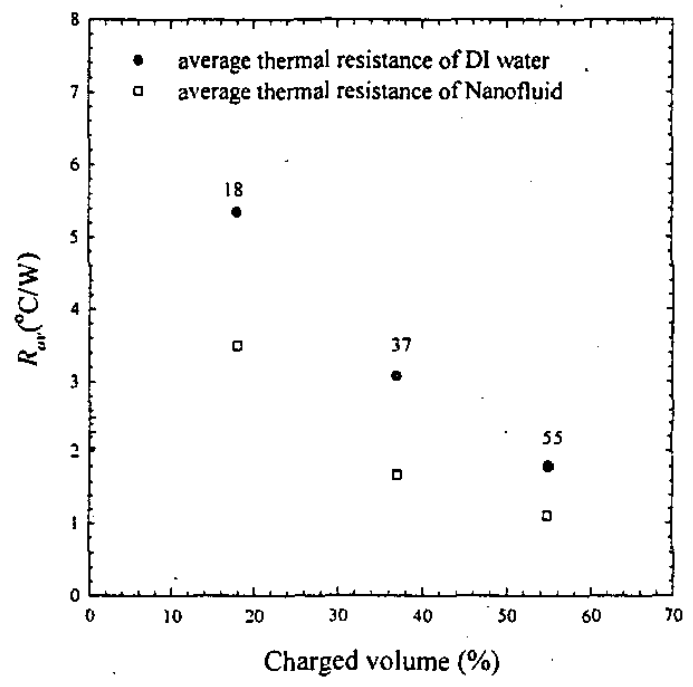

Figure 4 The thermal resistances compare between DJ water and nanofluid under different charge volume

thermal resistance can be achieved by DMHP with nano-gold particles.

As a result, the high heat spreading features of the new refrigerant have proved the potential as a substitute for conventional DI water.

\section{References}

H. C. Huang, C. P. Yin, Y. T. Ker and and T. F. Lin, (1998) Enhancement of boiling heat transfer in water through adding solid particles. The Eleventh International Symposium on 
Transport Phenomena, ISTP-11, no. 44. pp. 264-272.

H. T. Chien, D. S. Lee, P. P. Ding, S. L. Chiu, and P. H.

Chen, Disk-Shaped Miniature Heat Pipe (DMHP) with

Radiating Micro Grooves for a TO Can Laser Diode Package,

IEEE Transaction on Components and Packaging

Technologies. (2003 in press) 